\title{
Phytoglycogen to increase lutein solubility and its permeation through Caco- 2 monolayer
}

\author{
Hua Chen, Yuan Yao* \\ Department of Food Science, Purdue University, United States
}

\section{A R T I C L E I N F O}

\section{Keywords:}

Lutein

Phytoglycogen

Complexation

Solubility

Transepithelial permeation

\begin{abstract}
A B S T R A C T
Increasing the solubility of poorly water-soluble bioactives is essential to enhancing their bioavailability. The objective of this study was to evaluate the impact of phytoglycogen (PG) on water solubility of lutein and its transepithelial permeation across Caco-2 cell monolayers. Solid PG-LT complexes were prepared by combining an acetone solution of LT with an aqueous solution of PG under sonication, followed by centrifugation and vacuum drying of the supernatant as well as a further purification procedure. X-ray powder diffraction and differential scanning calorimetry showed negligible crystalline structure of LT in the PG-LT complex tested. The maximal water solubility of LT $(130.65 \mu \mathrm{g} / \mathrm{mL})$ occurred at the PG/LT combination ratio of 53.3/1, which was significantly higher than that of LT alone $(0.56 \mu \mathrm{g} / \mathrm{mL})$. Remarkably, LT, when complexed with PG, exhibited much-enhanced permeation through Caco-2 monolayer than LT alone, suggesting a potential role of PG to improve LT bioavailability. This study indicated that PG could be applied for the delivery of LT and possibly other hydrophobic ingredients.
\end{abstract}

\section{Introduction}

Lutein ( $\beta$, $\varepsilon$-carotene $3,3^{\prime}$-diol, $\mathrm{C}_{40} \mathrm{H}_{56} \mathrm{O}_{2}$ ) is a xanthophillic carotenoid, naturally occurring in a wide variety of fruits and vegetables, such as kale, collards, and spinach, comprising the characteristic yellow color (Fernández-Sevilla, Fernández, \& Grima, 2010). It is also present in some animal products such as egg yolk due to the plants eaten by the animal (Vishwanathan, 2010). LT selectively concentrates in the macula, and plays a fundamental role in the prevention of agerelated macular degeneration, cataracts, and other ocular disorders by acting as effective antioxidant and filter of high-energy blue light (Chew, SanGiovanni, Ferris, et al., 2013; Kijlstra, Tian, Kelly, \& Berendschot, 2012). In the US, LT is recognized as GRAS (generally recognized as safe) and allowed for inclusion into certain food applications (Bettler, Zimmer, Neuringer, \& DeRusso, 2010). It is commercially available as extract from marigold oleoresin and widely marketed as a dietary supplement to maintain eye health (Kalt, Hanneken, Milbury, \& Tremblay, 2010; Kawabata \& Tsuji, 2011). A diverse body of evidence has also accumulated indicating that LT may reduce the risk of coronary heart disease, stroke, atherosclerosis, lung cancer, and UV-induced skin damage (Han et al., 2015; Kim, Leite, Smyth, Clark, \& Fernandez, 2011; Li et al., 2012; Roberts, Green, \& Lewis, 2009; Satia, Littman, Slatore, Galanko, \& White, 2009; Shanmugasundaram \& Selvaraj, 2011).
However, applications of LT have been hindered by its poor water solubility, instability when exposed to light and heat, and low bioavailability (Arunkumar, Prashanth, \& Baskaran, 2013; Douroumis \& Fahr, 2012). To address the solubility limitations of LT, numerous lipid-based delivery systems have been exploited, such as liposomes (Tan et al., 2013), nanoemulsions (Murillo et al., 2016; Vishwanathan, 2010), solid lipid nanoparticles (Mitri, Shegokar, Gohla, Anselmi, \& Muller, 2011), nanostructured lipid carriers (Liu \& Wu, 2010), and self-emulsifying drug delivery systems (Shanmugam et al., 2011). For lipid-based systems, challenges may be associated with potential oxidative stress to LT and lipids upon heating over the preparation process, partial digestion of carrier lipids and thus reduced dissolution of LT during the gastrointestinal transit, low stability, limited scalability, and inconvenience of handling liquid forms. Undoubtedly, there exists a compelling need for non-lipid systems for LT delivery.

In this work, the feasibility of using phytoglycogen (PG) to improve the water solubility and transepithelial permeation of LT was evaluated. Phytoglycogen (PG) is a dendrimer-like $\alpha$-D-glucan generated by plant (Huang \& Yao, 2011; Wong et al., 2003). In PG, there is no long chain that connects individual clusters, indicative of a basic structural variation between PG and amylopectin in starch. The structural characteristics of PG allowed us to consider that the spacing among the glucan branches of PG particles would harbor and disperse insoluble

\footnotetext{
* Corresponding author at: 745 Agriculture Mall Drive, West Lafayette, IN 47907, United States.

E-mail address: yao1@purdue.edu (Y. Yao).
} 
small-molecule compounds such as LT.

\section{Materials and methods}

\subsection{Chemicals}

Lutein (purity $>$ 97\%) was purchased from Xi'an TianBen Bioengineering Co., Ltd. (Xi'an, China). All other chemicals for this study were of HPLC grade unless specified.

\subsection{Methods}

\subsubsection{Extraction of $P G$}

Dry kernels (320 g) of SU1 corn variety were grounded and soaked in ten times the weight of deionized water. The mixture was subjected to homogenization using a blender and the homogenates were passed through a 270 -mesh sieve. The fluid collected was adjusted to around $\mathrm{pH} 4.8$ and placed at $4{ }^{\circ} \mathrm{C}$ for $2 \mathrm{~h}$ to precipitate proteins. Thereafter, the extract was centrifuged $(10,000 \times g)$ repetitively until no precipitate was present. The supernatant collected was adjusted to $\mathrm{pH} 7.0$, and autoclaved at $121{ }^{\circ} \mathrm{C}$ for $50 \mathrm{~min}$. The insoluble proteinaceous material was removed using centrifugation, and 3 times the volume of ethanol were added to the supernatant to precipitate the polysaccharides. After centrifugation, the precipitate was re-suspended in ethanol and then centrifuged. This re-suspension and centrifugation procedure was repeated for 3 times. Thereafter, the bulk ethanol in the solid was removed using vacuum filtration, and the solid pellets were placed in a fume hood to remove residual ethanol. The dry solid collected was the PG material.

\subsubsection{Incorporation of phytoglycogen and lutein}

A $20 \mathrm{wt} \%$ dispersion of PG was prepared in sodium acetate buffer (50 mM, pH 6.8) by incubating at $70^{\circ} \mathrm{C}$ for $5 \mathrm{~min}$ with stirring. Accurately weighed amounts of LT crystals were suspended with acetone at concentrations of $80,40,30,20,15,10,8$, and $6 \mathrm{mg} / \mathrm{mL}$, respectively. The suspensions were allowed to stand for $5 \mathrm{~min}$ with stirring, and sonicated 3 times each for $30 \mathrm{~s}$ at power output 8 (Sonifier 150, Branson Ultrasonics Corp.). Subsequently, $1 \mathrm{~mL}$ aliquot from each LT dispersion was added dropwise into $4 \mathrm{~mL}$ PG dispersion with sonication (six 30-second bursts at setting 80 ) followed by vortexing for $1 \mathrm{~min}$. The resultant dispersions of different weight ratios of PG/LT $(10 / 1,20 / 1,26.7 / 1,40 / 1,53.3 / 1,80 / 1,100 / 1$ and 133.3/1) were subjected to solvent removal in a vacuum concentrator (SpeedVac, Thermo Scientific, USA) at $40{ }^{\circ} \mathrm{C}$. The solids thus generated were PG-LT complexes and termed as PG-LT-\#1S, with "1" indicating the initial incorporation of PG and LT and "S" indicating sonication. The above procedure was also performed in the absence of sonication to prepare PG-LT-\#1N, with "N" indicating non-sonication.

In a second step, the soluble portion of each PG-LT-\#1S material was isolated and termed as PG-LT-\#2. To achieve this, $20 \mathrm{mg} / \mathrm{mL}$ of each PG-LT-\#1S material was equilibrated in $\mathrm{ddH}_{2} \mathrm{O}$ in the dark with constant stirring for $4 \mathrm{~h}$ at room temperature $\left(22^{\circ} \mathrm{C}\right)$. Subsequently, the supernatant after centrifugation $(5000 \times g, 5 \mathrm{~min})$ was dried under vacuum (SpeedVac) to obtain PG-LT-\#2. As comparisons, physical mixtures were prepared by grinding the mixtures of various weight ratios of PG and LT in a mortar and pestle. All samples were stored in capped glass vials in the dark at room temperature.

\subsubsection{Lutein quantification}

For some LT solutions, LT concentration was determined using UV-vis spectrophotometer (DU 730, Beckman Coulter, USA) at $450 \mathrm{~nm}$. LT stock solution of $1.0 \mathrm{mg} / \mathrm{mL}$ was prepared with $80 \mathrm{vol} \%$ acetone and was serial diluted $(0.5-4.0 \mu \mathrm{g} / \mathrm{mL})$ using the same solvent to generate standard curve.

\subsubsection{Solubility of lutein-containing materials}

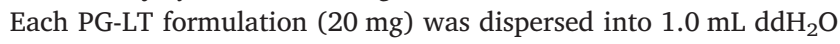
in a $2 \mathrm{~mL}$ microcentrifuge tube. The tube was wrapped with aluminum foil and vortexed for $3 \mathrm{~min}$, followed by equilibration at room temperature for $24 \mathrm{~h}$ on a bi-directional rotator (Lab-line 4631, Maxi Rotator, USA). Thereafter, the dispersion was centrifuged $(5000 \times g$, $5 \mathrm{~min}$ ) to remove insolubles, and $0.2 \mathrm{~mL}$ supernatant was withdrawn and added into $0.8 \mathrm{~mL}$ acetone. The mixture was vortexed for $1 \mathrm{~min}$ and centrifuged $(10,000 \times g, 5 \mathrm{~min})$ to separate PG precipitate; the amount of LT retained in the supernatant was quantified using the abovementioned method and considered as water-soluble. The total amount of soluble LT (based on $20 \mathrm{mg}$ solid and $1.0 \mathrm{~mL} \mathrm{H}_{2} \mathrm{O}$ ) was presented as the solubility. All experiments were carried out in triplicate.

\subsubsection{Solid state characterization of lutein-containing materials}

2.2.5.1. DSC analysis. Thermal characterization of LT alone and PG-LT formulations was conducted using differential scanning calorimetry (DSC, TA instruments, USA). For each solid, about $5 \pm 1 \mathrm{mg}$ were accurately weighed and placed in hermetically sealed aluminum pans and scanned at a rate of $20^{\circ} \mathrm{C} / \mathrm{min}$ under nitrogen from 30 to $250{ }^{\circ} \mathrm{C}$. An empty pan was used as reference.

2.2.5.2. X-ray powder diffraction analysis. The crystalline pattern of LT in various solid materials was evaluated using X-ray powder diffractometer (XRD-6000, Shimadzu, North America) equipped with a Bragg-Brentano optical setup. Nickel filtered $\mathrm{Cu} \mathrm{K \alpha}$ radiation $(\lambda=1.5406 \AA)$ was used with a long fine focus X-ray tube. Samples were scanned at $2 \theta$ of $3^{\circ}-35^{\circ}$ with a speed of $8^{\circ}(2 \theta) / \mathrm{min}$ at $40 \mathrm{kV}$ and $30 \mathrm{~mA}$. The diffraction patterns of LT alone, PG, physical mixtures of LT and PG, and the PG-LT complexes were recorded respectively.

\subsubsection{Determination of Caco-2 transepithelial permeation of lutein}

2.2.6.1. Preparation of lutein alone test solutions. Stock solutions of LT alone with concentrations of 3,6 , and $12 \mathrm{mM}$ were prepared individually with 50 vol\% ethanol in HBSS ( $\mathrm{pH}$ 6.5) and then diluted to 30,60 , and $120 \mu \mathrm{M}$ respectively using HBSS (with final ethanol concentration of $0.5 \mathrm{vol} \%$ ). All LT test solutions were homogenized at $11600 \mathrm{rpm}$ for $5 \mathrm{~min}$ using a high-speed homogenizer (Ultra-Turrax T 25, IKA). Both TEER values and cell viability were found to be unaffected by the 0.5 vol\% ethanolic HBSS. It was noted that $120 \mu \mathrm{M}$ LT dispersion led to colored precipitate upon equilibration at room temperature, thus the use of this concentration was discontinued in subsequent experiments.

2.2.6.2. Preparation of phytoglycogen-lutein complex test solutions. For Caco-2 permeation test, PG-LT-\#2 solid prepared from PG-LT-\#1S (53.3:1) was used due to its high solubility. The PG-LT-\#2 solid was dispersed and diluted with HBSS (pH 6.5 with 0.5 vol\% ethanol) to prepare a series of working solutions containing 30, 60, and $120 \mu \mathrm{M}$ soluble LT, respectively. To ensure the uniformity of these preparations, all PG-LT-\#2 test solutions were homogenized at $11600 \mathrm{rpm}$ for $5 \mathrm{~min}$. All the test preparations were prepared right before Caco- 2 permeation test, and maintained at $37^{\circ} \mathrm{C}$.

2.2.6.3. Cell culture. The Caco-2 BBE cell line (ATCC, Rockville, MD) was cultured with DMEM with $10 \%$ fetal calf serum, $1 \%$ sodium pyruvate, and $1 \%$ penicillin in a humidified incubator $\left(5 \% \mathrm{CO}_{2}\right)$ at $37{ }^{\circ} \mathrm{C}$ to $80 \%$ confluency. Cells in passages between 57 and 68 were used. For the permeation studies, cells were harvested and seeded onto $12 \mathrm{~mm}$ i.d. Transwell inserts (polycarbonate membrane, $0.4 \mu \mathrm{m}$ pore size, insert membrane growth area: $1.12 \mathrm{~cm}^{2}$, Corning Costar Corp. NY, USA) of 12 -well plates at a density of $3.5 \times 10^{5} \mathrm{cells} / \mathrm{cm}^{2}$. The culture medium $(0.5 \mathrm{~mL}$ in the insert and $1.5 \mathrm{~mL}$ in the well) was replaced every $48 \mathrm{~h}$ for the first 6 days and every $24 \mathrm{~h}$ thereafter for a total of 22 days. The integrity of the monolayers was evaluated routinely by measuring the transepithelial electric resistance (TEER) with Millicell- 
ERS equipment (Millipore, USA). Only monolayers with a TEER of $>350 \Omega \mathrm{cm}^{2}$ were used. TEER values were measured prior to and during the experiment to confirm the integrity of monolayers.

2.2.6.4. Cell viability. The impact of LT alone and PG-LT solutions on the viability of Caco-2 cells was evaluated by the colorimetric MTT (3(4,5-dimethylthiazol-2-yl)-2,5-diphenyltetrazolium bromide) reduction assay. Cells at the amount of $3 \times 10^{5}$ were seeded in 96-well plate (Corning Costar, USA) in $100 \mu \mathrm{L}$ of cell culture medium. The cells were then cultured under the same condition as the monolayer preparation for $96 \mathrm{~h}$ before use. Immediately prior to each experiment, the medium was removed, and $100 \mu \mathrm{L}$ of LT alone solutions (30 and $60 \mu \mathrm{M}$ ) and PGLT preparations (equivalent to 30,60 , and $120 \mu \mathrm{M}$ soluble LT) were added to individual well respectively. HBSS without any form of LT served as a control. After $2 \mathrm{~h}$ of incubation, $10 \mu \mathrm{L}$ of $5 \mathrm{mg} / \mathrm{mL}$ MTT solution (in PBS) was added to each well; the cells were incubated for another $2 \mathrm{~h}\left(37^{\circ} \mathrm{C}, 5 \% \mathrm{CO}_{2}\right)$ to allow MTT to be metabolized. Then the medium was removed and the formazan crystals (product of MTT metabolism) were dissolved in $300 \mu \mathrm{L}$ DMSO. The absorbance values were read at $595 \mathrm{~nm}$ on a microplate reader (iMark, Bio-Rad Instruments, Inc., USA). The percent cell viability was calculated using the equations below:

Cell viability $(\%)=\frac{\mathrm{LT}_{\text {signal-background }}}{\mathrm{HBSS}_{\text {control-background }}} \times 100$

In Eq. (1), the value of LT signal-background was calculated as:

$\mathrm{LT}_{\text {signal-background }}=$ absorbance of LT solution - absorbance of DMSO blank

The value of HBSS control-background was calculated as:

HBSS $_{\text {control-background }}=$ absorbance of HBSS control

- absorbance of DMSO blank

2.2.6.5. Stability of lutein in HBSS. Stability of LT upon exposure to HBSS medium was studied. The test was carried out in transwell by incubating test solutions of LT alone and PG-LT in cell-free HBSS at pH 6.5 (as in the apical side) and 7.4 (as in the basolateral side) respectively in a shaker bath for $2 \mathrm{~h}\left(37^{\circ} \mathrm{C}, 60 \mathrm{rpm}\right)$, followed by measuring LT concentration using UV-vis spectrophotometer at $450 \mathrm{~nm}$. Stability of LT in the HBSS medium was presented as the percentage of LT remained soluble after incubation for $2 \mathrm{~h}$.

2.2.6.6. Cellular uptake and transepithelial permeation of lutein. Spent medium from the apical and basolateral sides were aspirated before the experiment. Then the monolayers were rinsed twice with warm PBS $\left(\mathrm{pH} 7.4,37^{\circ} \mathrm{C}\right)$ and incubated twice with HBSS (pH 7.4) each for $30 \mathrm{~min}$ in a $\mathrm{CO}_{2}$ incubator at $37^{\circ} \mathrm{C}$. Thereafter, the medium was removed by aspiration, followed by supplementation of $0.5 \mathrm{~mL}$ aliquot of test solutions ( 30 and $60 \mu \mathrm{M}$ LT of LT alone solutions, and 30,60 , and $120 \mu \mathrm{M} \mathrm{LT}$ of PG-LT complex solutions) respectively to the apical compartment and $1.5 \mathrm{~mL}$ HBSS ( $0.5 \mathrm{vol} \%$ ethanol, $\mathrm{pH} 7.4$ ) to the basolateral compartment. For the control, $0.5 \mathrm{~mL}$ HBSS $(0.5$ vol\% ethanol, pH 6.5) was added to the apical side, while the basolateral side received $1.5 \mathrm{~mL}$ HBSS ( $0.5 \mathrm{vol} \%$ ethanol, $\mathrm{pH} 7.4)$. Each test solution was loaded in triplicate, and the cultures were incubated in a $\mathrm{CO}_{2}$ incubator for $2 \mathrm{~h}$.

After the incubation, the apical and the basolateral solutions were sampled respectively. Thereafter, each insert was rinsed three times with $0.5 \mathrm{~mL}$ ice-cold PBS and then incubated for $30 \mathrm{~min}$ with methanol containing $0.5 \mathrm{vol} \%$ acetic acid. The cells were scraped off and the resultant mixture was sonicated for $10 \mathrm{~min}$ at room temperature and centrifuged at $1600 \times g$ for $5 \mathrm{~min}$. The precipitate was rinsed with $1 \mathrm{~mL}$ methanol and centrifuged. The procedure was repeated twice and all the supernatants were combined as cellular extracts. The apical and basolateral solutions and the cellular extracts were evaporated to dryness under vacuum. The residues were reconstituted in $250 \mu \mathrm{L}$ methanol for HPLC analysis.

TEER values were monitored before and after the permeation experiments. It was found that the $2 \mathrm{~h}$ incubation with the test solutions did not adversely affect TEER values. All measurements were performed in triplicate.

2.2.6.7. HPLC analysis of lutein. The amounts of LT in the apical and basolateral medium and the cellular extracts (nmol/well) were determined using a Supelcosil LC-18-DB reverse phase column $(4.6 \times 150 \mathrm{~mm}$, i.d., $3 \mu \mathrm{m})$ and a model 998 photodiode array (PDA) detector at $450 \mathrm{~nm}$. The mobile phase consisted of $95 \mathrm{vol} \%$ of reservoir A (acetonitrile: methanol $=7: 1, \mathrm{v} / \mathrm{v}$ ) and $5 \mathrm{vol} \%$ of reservoir $\mathrm{B}$ (dichloromethane). An isocratic elution method was performed at ambient temperature with a flow rate of $1.0 \mathrm{~mL} / \mathrm{min}$ and injection volume was $20 \mu \mathrm{L}$. The calibration curve was linear $\left(r^{2}=0.999\right)$ over the concentration range of $0.25-100 \mu \mathrm{g} / \mathrm{mL}$. Chromatographic data were acquired using Agilent ChemStation, version B.01.03.

\subsubsection{Statistical analysis}

Most results were expressed as mean \pm standard deviation. Comparisons among individual groups were conducted at a $95 \%$ confidence level $(P \leq 0.05)$ using one-way ANOVA followed by Tukey's test.

\section{Results and discussion}

\subsection{Phytoglycogen/lutein ratio affected lutein solubility}

Varying amounts of LT were incorporated with PG with or without sonication. As shown in Fig. 1, the use of sonication effectively increased the solubility of LT. For all formulations at PG/LT ratio from $20 / 1$ to $133.3 / 1$, LT solubility for each PG-LT-\#1S (with sonication) was significantly higher than its PG-LT-\#1N (no sonication, $P<0.05$ ) and PM (physical mixing of PG and LT, $P<0.05$ ) counterparts. However, there appeared to be no significant difference $(P>0.05)$ in LT solubility between PG-LT-\#1S and PG-LT-\#1N for PG/LT ratio of 10/1, probably due the pronounced abundance of LT fed.

As expected, the feed ratio of PG to LT affected LT solubility, with the highest LT feeding (PG/LT of 10/1) yielding the lowest LT solubility. A decrease in LT feeding that corresponded to an increase of PG/LT ratio from $26.7 / 1$ to $53.3 / 1$ resulted in a significant increase in LT solubility $(P<0.05)$, reaching the maximal level at PG/LT ratio of $53.3 / 1$, showing $93.69 \mu \mathrm{g} / \mathrm{mL}$ for PG-LT-\#1S and $43.92 \mu \mathrm{g} / \mathrm{mL}$ for PG-LT-\#1N. Nevertheless, further increase of PG/LT ratio from 53.3/1 to $133.3 / 1$ caused a gradual but significant reduction of LT solubility for most formulations $(P<0.05)$.

It was considered that the appearance of a peak solubility value at a specific PG/LT ratio was associated with the abundance and growth of LT crystal nuclei. It was possible to speculate that, at the initial stage of PG-LT mixing, LT crystal nuclei were generated from amorphous molecules, initiating further crystallization. At high LT doses (e.g. PG/LT of 10/1), the amount of LT crystal nuclei was abundant, and therefore the partitioning of amorphous LT molecules (from the acetone solution) onto the nuclei (in the water-acetone mixture) exceeded their association with PG particulates. At lower LT doses (i.e. high PG/LT ratios), the association of amorphous LT to PG was enhanced since lower amount of LT nuclei were readily available to promote crystallization, thus leading to higher LT solubility with the highest solubility realized at PG/LT of 53.3/1. When the LT dose was further reduced (i.e. increasing PG/LT ratio from 53.3/1 to $133.3 / 1$ ), a decrease of LT solubility was observed, presumably due to the reduction in the net amount of LT available for incorporation with PG. In general, the results showed that the LT feeding dose had a major impact on the solubility of LT. 


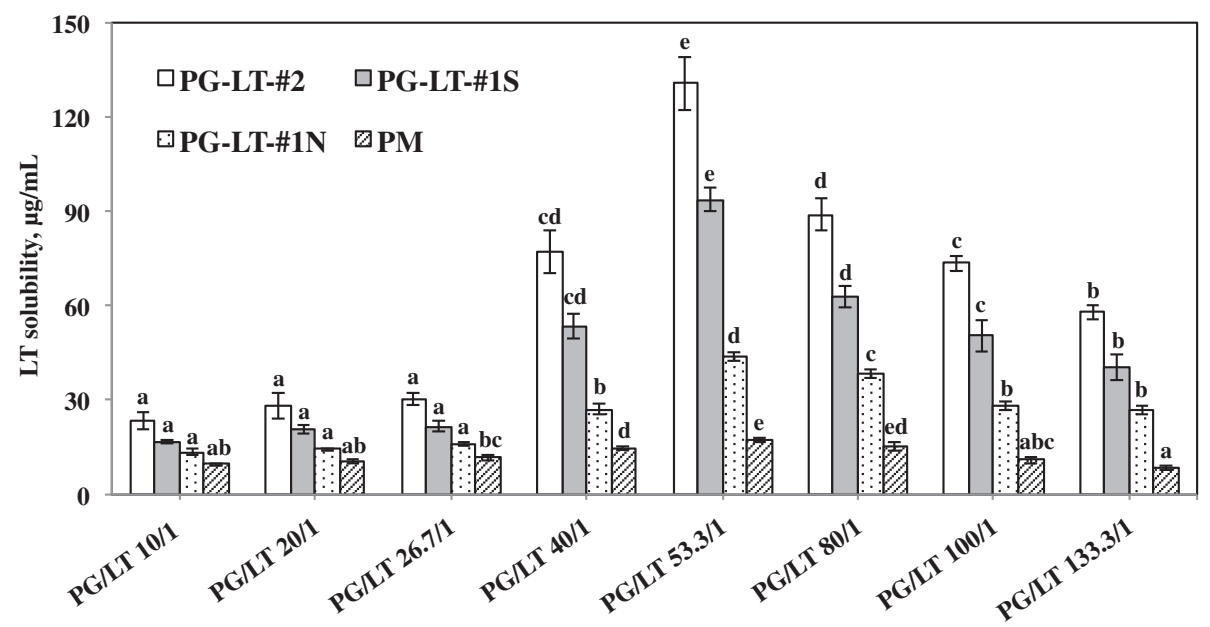

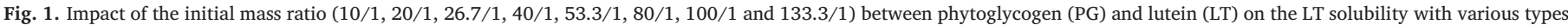

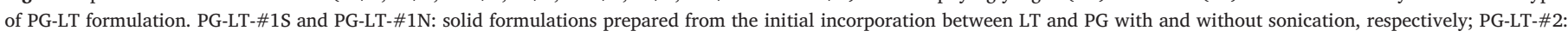

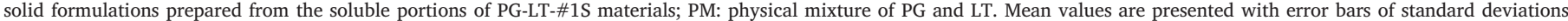
$(\mathrm{n}=3)$. Within each type of formulation (i.e. PG-LT-\#2, PG-LT-\#1S, PG-LT-\#1N, and PM), mean values that do not share a common letter differ significantly $(P<0.05)$.

For each PG/LT ratio (from 10:1 to 133.3:1), the amount of soluble LT for PG-LT-\#2 was substantially higher than that for other formulations (PG-LT-\#1S, PG-LT-\#1N, and PM) (Fig. 1). This was understandable because each vacuum-dried PG-LT-\#1N or -\#1S solid formulation was a heterogeneous composite with LT existed in two forms: (1) those associated with PG particulates and thus were water-soluble, and (2) those formed crystallites and thus were insoluble. For the preparation of PG-LT-\#2 solids, LT crystallites were mostly eliminated, thus increasing the portion of soluble LT. For example, at the PG/LT ratio of 53.3/1, the LT solubility of PG-LT-\#2 was $130.65 \mu \mathrm{g} / \mathrm{mL}$, much higher than that of PG-LT-\#1S (93.69 $\mu \mathrm{g} / \mathrm{mL}), \mathrm{LG}-\mathrm{LT}-\# 1 \mathrm{~N}(43.92 \mu \mathrm{g}$ / $\mathrm{mL})$, and PM $(17.36 \mu \mathrm{g} / \mathrm{mL})$.

\subsection{Incorporation with phytoglycogen reduced lutein crystallinity}

\subsubsection{DSC analysis}

DSC was used to evaluate the capability of PG to reduce the ordered structure of LT. As shown in Fig. 2, PG displayed no distinct endotherm over the entire range of the temperature examined $\left(50-250{ }^{\circ} \mathrm{C}\right)$. In contrast, LT alone showed a prominent melting endothermic peak with melting temperature $\left(T_{\mathrm{m}}\right)$ at $180-190{ }^{\circ} \mathrm{C}$, which was in compliance with

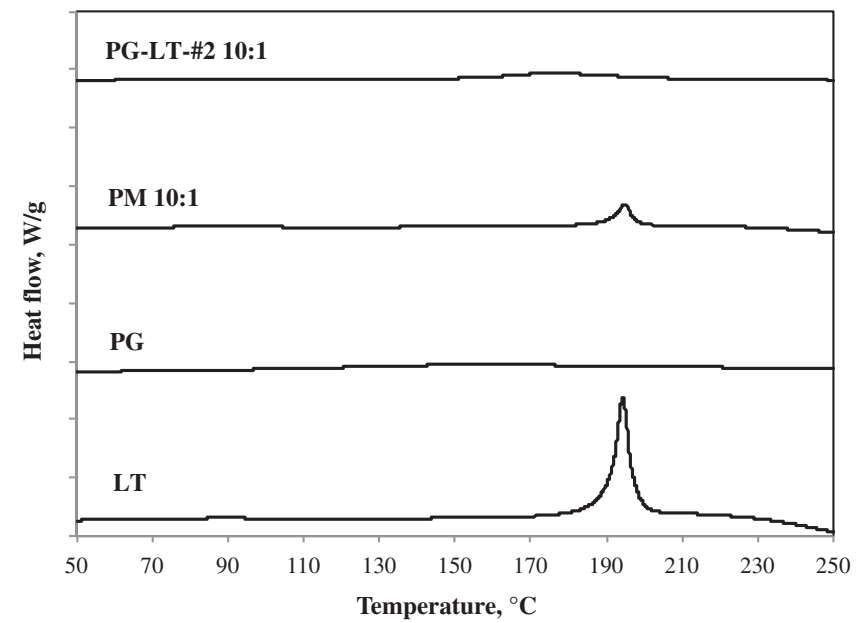

Fig. 2. DSC thermograms of lutein (LT)-containing solids. LT: original LT solid purchased; PG: phytoglycogen; PM 10:1: physical mixture between PG and LT at a mass ratio of 10:1; PG-LT-\#2 10:1: solid prepared from the soluble portion of the PG-LT-\#1S material that was prepared with a mass ratio of 10:1 between PG and LT. For ease of comparison, results are vertically displaced by arbitrary amounts. earlier reports (Hu, Lin, Liu, Li, \& Zhao, 2012; Xia, Hu, Jin, Zhao, \& Liang, 2012). The melting endotherm of LT was evident in the case of physical mixture of PG and LT at a ratio of 10/1 (PM 10:1), implying a simple diluting effect of LT by PG. In comparison, PG-LT-\#2 10:1 presented an absence of melting endotherm peak specific to LT, even through a broad and low bump could be seen roughly at $150-210{ }^{\circ} \mathrm{C}$. These observations indicated a substantial reduction of LT ordered structure upon association with PG.

\subsubsection{XRD analysis}

Fig. 3 compares the X-ray crystallogram of PG-LT-\#2 10:1 with that of LT, PG, and physical mixture of PG and LT. In general, LT exhibited characteristic peaks over the range of $5-25^{\circ}$, indicating its highly crystalline nature. PG was amorphous since no obvious diffraction peaks appeared in its crystallogram. For PM 10:1, the characteristic peaks of LT crystalline structure were reduced yet evident, showing that LT crystallites were diluted with PG. In comparison, PG-LT-\#2 10:1 showed an absence of characteristic LT peaks, suggesting that the crystallinity of LT was suppressed after incorporation with PG.

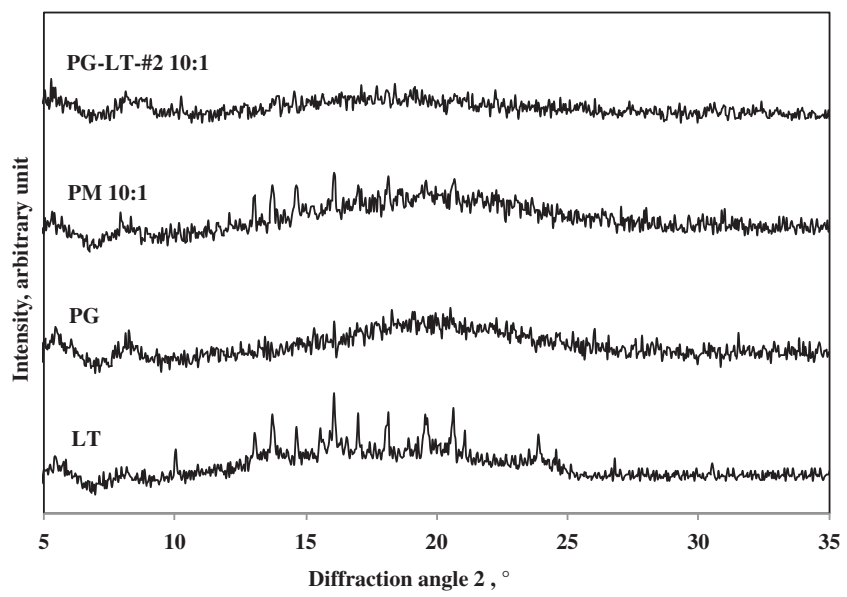

Fig. 3. X-ray powder diffraction crystallograms of lutein (LT)-containing solids. LT: original LT solid purchased; PG: phytoglycogen; PM 10:1: physical mixture between PG and LT at a mass ratio of 10:1; PG-LT-\#2 10:1: solid prepared from the soluble portion of the PG-LT-\#1S material that was prepared with a mass ratio of 10:1 between PG and LT. For ease of comparison, results are vertically displaced by arbitrary amounts. 


\subsection{Cell viability affected by lutein concentration}

The impact of LT alone and PG-LT solutions on the viability of Caco2 cells was examined using MTT assay. As indicated in the Fig. S1 of Supplemental material, a high cell viability of $>93 \%$ was observed in all cases, indicating that the LT alone and PG-LT preparations were not cytotoxic to the Caco-2 cells with LT concentration of up to $120 \mu \mathrm{M}$. According to a previous study, the $\mathrm{IC}_{50}$ value of LT in Caco- 2 cell model was $265.4 \mu \mathrm{g} / \mathrm{mL}$ (Daly et al., 2010), which was well above the highest supplementation of PG-LT ( $120 \mu \mathrm{M}$ or $68.26 \mu \mathrm{g} / \mathrm{mL})$ used in our study.

The non-cytotoxic effect of the PG-LT preparation indicated the high safety of PG as a delivery vehicle. Niamprem, Rujivipat, and Tiyaboonchai (2014) tested the toxicity of an LT-loaded self-nanoemulsifying drug delivery system (SNEDDS). They found that at LT concentration of $30 \mu \mathrm{g} / \mathrm{mL}$ the Caco-2 cell viability decreased to $40 \%$, which was ascribed to the potential damage to cell membranes caused by surfactants. Conceivably, the use of PG showed a superior safety profile over SNEDDS in delivering lutein.

\subsection{Stability of lutein in HBSS at different $p H$}

The retention of LT in the cell-free HBSS media (pH 6.5 and 7.4, $37^{\circ} \mathrm{C}$ ) delivered either as LT alone or with PG-LT complex was determined after $2 \mathrm{~h}$. Fig. 4 shows that the percentage of LT retention ranged from $91.0 \%$ to $97.5 \%$, suggesting the high stability of LT either alone or incorporated with PG. In contrast, the work of Chitchumroonchokchai, Schwartz, and Failla (2004) showed that alltrans-LT from digested spinach meals declined with only $75 \%$ remaining after $16 \mathrm{~h}$ of incubation in the cell-free environment, accompanied by the simultaneous appearance of an equivalent quantity of 13-cis-LT. This suggests that the stability of LT was associated with the duration of incubation and possibly the presence of other components. In general, the LT solutions and incubation conditions used in this study did not lead to appreciable LT reduction.

\subsection{Uptake and transcellular permeation of lutein with Caco-2 cell monolayer}

For LT alone and PG-LT, uptake of LT by Caco-2 cell monolayer increased in a dose-dependent pattern, and PG-LT was able to deliver substantially higher amount of LT to and across the cell monolayer than LT alone at the same LT concentration (Fig. 5). At the initial loading of $30 \mu \mathrm{M} \mathrm{LT}$ ( $15 \mathrm{nmol} /$ well), cellular uptake of LT delivered by PG-LT ( $3.72 \mathrm{nmol} /$ well) was much higher than that delivered by LT alone

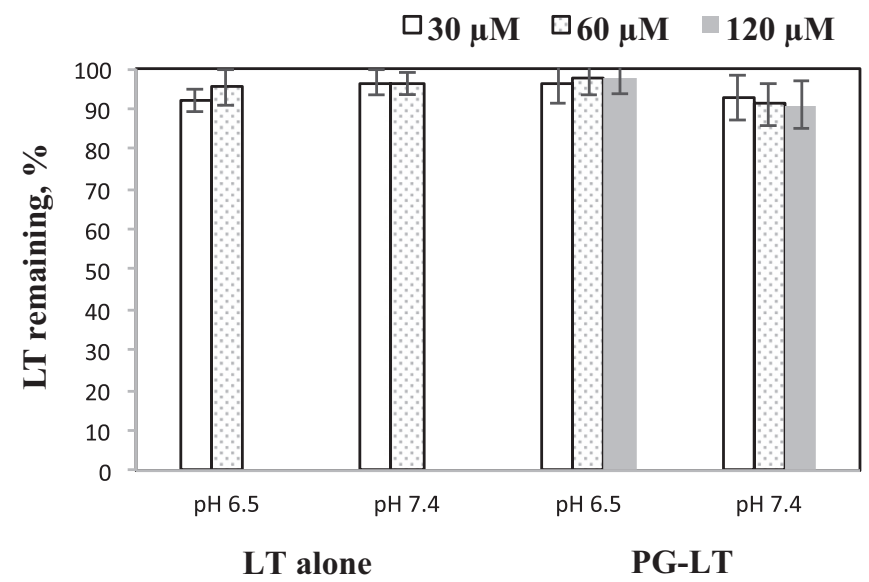

Fig. 4. Stability of lutein (LT) solution in cell-free HBSS after a $2 \mathrm{~h}$ incubation period. The testing results of LT concentrations of 30,60 , and $120 \mu \mathrm{M}$ are presented. LT alone: solutions prepared from LT solid; PG-LT: solutions prepared from a PG-LT-\#2 complex solid. Stability values are presented as \% LT retained after $2 \mathrm{~h}$ incubation. Data are presented as means with error bars of standard deviations $(n=3)$.

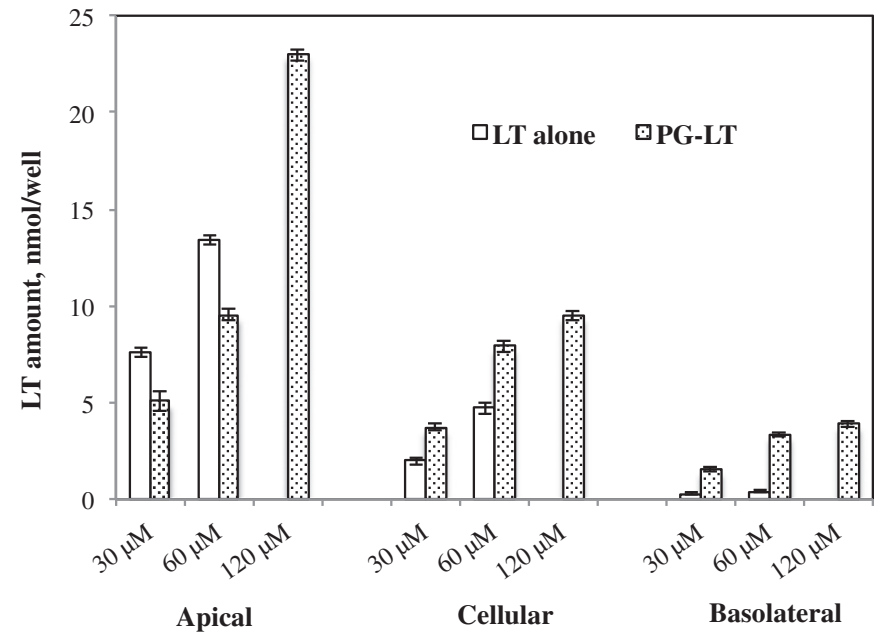

Fig. 5. Effect of lutein (LT) formulation and dose level on the cumulative amount of LT at the apical and basolateral side and in the cellular extract after the $2 \mathrm{~h}$ incubation. Labels of 30,60 , and $120 \mu \mathrm{M}$ indicate the concentrations of LT preparations loaded in the apical compartment, corresponding to initial LT loading of 15, 30, and $60 \mathrm{nmol} / \mathrm{well}$, respectively. LT alone: solutions prepared from LT solid; PG-LT: solutions prepared from a PG-LT-\#2 complex solid. Data are presented as means with error bars of standard deviations $(\mathrm{n}=3)$.

(1.98 nmol/well). At the highest LT dose of $120 \mu \mathrm{M}(60 \mathrm{nmol} /$ well $)$, which was impossible for LT alone to reach due to its inherent solubility constraint, PG-LT delivered $9.5 \mathrm{nmol} /$ well to the cells after $2 \mathrm{~h}$, accounting for $15.8 \%$ of the initial LT amount in the apical chamber.

Our findings indicated the potential of PG to increase the cellular uptake of LT. Previous study by O'Sullivan, Ryan, \& O'Brien (2007) found that the percentage uptake of LT at varying treatment doses ranged from 2.8 to $9.7 \%$ of the original LT amount after a $16 \mathrm{~h}$ incubation. In general, the varying and relatively low cellular accumulation of micellar LT was partly due to the differences in the carrier, composition, number, and size of micelles, and the poor solubility of LT (O'Sullivan et al., 2007; Chitchumroonchokchai et al., 2004; LornejadSchäfer, Lambert, Breithaupt, Biesalski, \& Frank, 2007; Niamprem et al., 2014). In our study, when PG-LT delivered LT at an initial level of $30 \mathrm{nmol} /$ well $(60 \mu \mathrm{M})$ on the apical side, the cellular uptake was $7.9 \mathrm{nmol} /$ well (Fig. 5), showing a very high percentage (26.3\%).

As shown by Fig. 5, LT permeation (i.e. LT received by basolateral compartment) was enhanced with an increasing loading dose of LT at the apical side. In addition, LT delivered by PG-LT complexes showed significantly higher permeation than that by LT alone at each dose level $(P<0.05)$. At $30 \mu \mathrm{M}(15 \mathrm{nmol} /$ well $)$ and $60 \mu \mathrm{M}(30 \mathrm{nmol} /$ well $)$ dose levels, the LT amount at the basolateral side was 1.57 and $3.36 \mathrm{nmol} /$ well respectively for PG-LT formulations, in comparison with 0.27 and $0.41 \mathrm{nmol} /$ well respectively for LT alone. For PG-LT, the LT amount at the basolateral side may reach $3.91 \mathrm{nmol} /$ well with an initial apical loading of $120 \mu \mathrm{M}(60 \mathrm{nmol} /$ well).

Fig. 6 conceptually compares the initial apical loading of LT alone (Fig. 6A) and PG-LT (Fig. 6B) with the same LT amount. Caco-2 cellular uptake and permeation results (Fig. 5) supported the assumption that the association of LT and PG exerted no adverse effect on the release of LT from PG particulates. The effective release of LT from PG-LT could be attributed to the physical interactions between individual LT molecules and the surface of PG particulates (Fig. 6B). We speculate that such physical interactions originated from the hydrophobic interactions between LT molecules and the external linear segments of PG glucan chains, similar to that between lipid and amylopectin (Villwock, Eliasson, Silverio, \& BeMiller, 1999).

Furthermore, we considered that the LT-glucan chain interaction would induce an increase of the hydrophobicity of the PG particulates, thereby enhancing their interactions with the cell membrane at the apical side. This would increase the local concentration of amorphous 


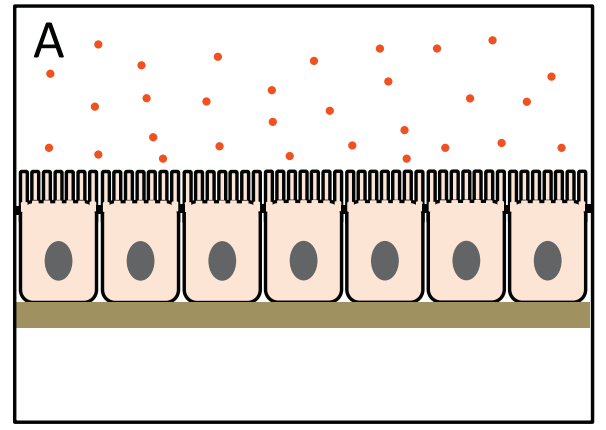

Caco-2 cell of a monolayer

\section{$\therefore \quad$ Dispersed lutein molecules}

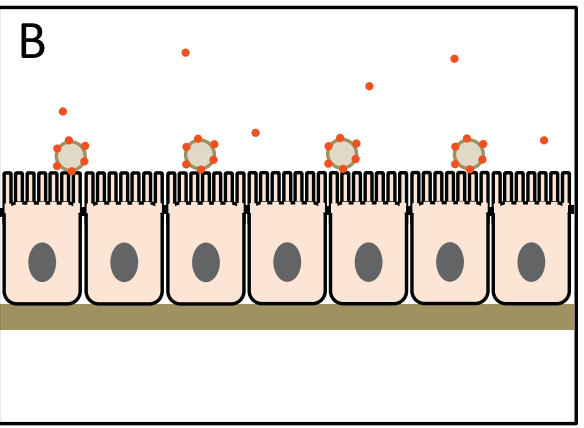

PG simplified schematic

\section{PG attached with lutein molecules}

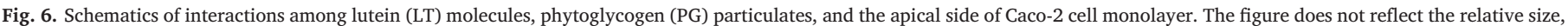
shape, and configuration of lutein molecules, PG particulates, and cells.

LT at the surface of cell membrane without causing the crystallization of LT molecules (Fig. 6B). In contrast, such a local enrichment of amorphous LT molecules might hardly occur in the case of the LT alone solutions (Fig. 6A). Local enrichment of LT would substantially increase its cellular uptake and permeation. As shown in Fig. 5, at an initial apical LT loading of $60 \mathrm{mM}(30 \mathrm{nmol} /$ well $)$, the cellular uptake and permeation to basolateral side were 7.9 and $3.36 \mathrm{nmol} /$ well respectively for PG-LT, and 4.71 and $0.41 \mathrm{nmol} /$ well respectively for LT alone.

For the PG-LT solutions, an increment of initial apical LT concentration from 60 to $120 \mu \mathrm{M}$ led to enhanced LT cellular uptake and basolateral permeation; however, neither uptake nor permeation was proportional to the concentration of LT loaded at the apical side (Fig. 5). Rather, there appeared to be a limit with the effect of the PG-LT loading dose on LT uptake and permeation, presumably due to a potential saturation of LT within the cell monolayer.

\section{Conclusions}

This study found that LT could be incorporated with PG to achieve enhanced water solubility and epithelial permeation. The use of sonication promoted the association between LT and PG, and thus increasing the LT solubility. The ratio of PG/LT proved to be a factor for LT solubility improvement, with PG/LT of 53.3/1 correlating with the highest LT solubility. DSC and X-ray powder diffraction analysis confirmed that PG-LT incorporation effectively reduced the ordered and crystalline structure of LT. The substantial enhancement of the in vitro cellular uptake and permeation of LT was probably ascribed to the local enrichment of amorphous LT at the surface of the apical cell membrane with PG particulates as delivering vehicles. PG is a naturally occurring carbohydrate polymer derived from crops, and its use to improve the solubility and bioavailability of hydrophobic active ingredients may have considerable implications to the food industry.

Supplementary data to this article can be found online at http://dx. doi.org/10.1016/j.foodres.2017.04.021.

\section{Acknowledgements}

The National Science Foundation grant No. 1006301 to Yuan Yao and Ganesan Narsimhan provided the financial support for this study. We also appreciate the assistance by Jingfan Chen in the manuscript preparation.

\section{References}

Arunkumar, R., Prashanth, K. V. H., \& Baskaran, V. (2013). Promising interaction between nanoencapsulated lutein with low molecular weight chitosan: Characterization and bioavailability of lutein in vitro and in vivo. Food Chemistry, 141(1), 327-337.

Bettler, J., Zimmer, J. P., Neuringer, M., \& DeRusso, P. A. (2010). Serum lutein concentrations in healthy term infants fed human milk or infant formula with lutein. European Journal of Nutrition, 49(1), 45-51.

Chew, E. Y., SanGiovanni, J. P., Ferris, F., et al. (2013). Lutein/zeaxanthin for the treatment of age-related cataract: AREDS2 randomized trial report no. 4. JAMA Ophthalmology, 131(7), 843-850.

Chitchumroonchokchai, C., Schwartz, S. J., \& Failla, M. L. (2004). Assessment of lutein bioavailability from meals and a supplement using simulated digestion and Caco-2 human intestinal cells. The Journal of Nutrition, 134(9), 2280-2286.

Daly, T., Ryan, E., Aherne, S. A., O'Grady, M. N., Hayes, J., Allen, P., Kerry, J. P., \& O'Brien, N. M. (2010). Bioactivity of ellagic acid-, lutein- or sesamol-enriched meat patties assessed using an in vitro digestion and Caco-2 cell model system. Food Research International, 43(3), 753-760.

Douroumis, D., \& Fahr, A. (2012). Drug delivery strategies for poorly water-soluble drugs. John Wiley \& Sons.

Fernández-Sevilla, J. M., Fernández, F. A., \& Grima, E. M. (2010). Biotechnological production of lutein and its applications. Applied Microbiology and Biotechnology, 86(1), 27-40.

Han, H., Cui, W., Wang, L., Xiong, Y., Liu, L., Sun, X., \& Hao, L. (2015). Lutein prevents high fat diet-induced atherosclerosis in ApoE-deficient mice by inhibiting NADPH oxidase and increasing PPAR expression. Lipids, 50(3), 261-273.

Hu, D., Lin, C., Liu, L., Li, S., \& Zhao, Y. (2012). Preparation, characterization, and in vitro release investigation of lutein zein nanoparticles via solution enhanced dispersion by supercritical fluids. Journal of Food Engineering, 109(3), 545-552.

Huang, L., \& Yao, Y. (2011). Particulate structure of phytoglycogen nanoparticles probed using amyloglucosidase. Carbohydrate Polymers, 83(4), 1665-1671.

Kalt, W., Hanneken, A., Milbury, P., \& Tremblay, F. (2010). Recent research on polyphenolics in vision and eye health $\dagger$. Journal of Agricultural and Food Chemistry, 58(7), 4001-4007.

Kawabata, F., \& Tsuji, T. (2011). Effects of dietary supplementation with a combination of fish oil, bilberry extract, and lutein on subjective symptoms of asthenopia in humans. Biomedical Research, 32(6), 387-393.

Kijlstra, A., Tian, Y., Kelly, E. R., \& Berendschot, T. T. J. M. (2012). Lutein: More than just a filter for blue light. Progress in Retinal and Eye Research, 31(4), 303-315.

Kim, J. E., Leite, J. O., Smyth, J. A., Clark, R. M., \& Fernandez, M. L. (2011). A luteinenriched diet prevents cholesterol accumulation and decreases oxidized LDL and inflammatory cytokines in the aorta of guinea pigs. The Journal of Nutrition, 141(8), 1458-1463.

Li, S.-Y., Yang, D., Fu, Z. J., Woo, T., Wong, D., \& Lo, A. C. Y. (2012). Lutein enhances survival and reduces neuronal damage in a mouse model of ischemic stroke. Neurobiology of Disease, 45(1), 624-632.

Liu, C.-H., \& Wu, C.-T. (2010). Optimization of nanostructured lipid carriers for lutein delivery. Colloids and Surfaces A: Physicochemical and Engineering Aspects, 353(2), $149-156$.

Lornejad-Schäfer, M. R., Lambert, C., Breithaupt, D. E., Biesalski, H. K., \& Frank, J. (2007). Solubility, uptake and biocompatibility of lutein and zeaxanthin delivered to cultured human retinal pigment epithelial cells in tween 40 micelles. European Journal of Nutrition, 46(2), 79-86.

Mitri, K., Shegokar, R., Gohla, S., Anselmi, C., \& Muller, R. H. (2011). Lipid nanocarriers for dermal delivery of lutein: Preparation, characterization, stability and performance. International Journal of Pharmaceutics, 414(1), 267-275. 
Murillo, A. G., Norris, G. H., DiMarco, D. M., Hu, S., Luo, Y., Blesso, C. N., \& Fernandez, M.-L. (2016). A nano-emulsion of lutein is more effective than regular lutein in reducing cholesterol-induced liver injury in guinea pigs. The FASEB Journal, 30(1), 913. 2.

Niamprem, P., Rujivipat, S., \& Tiyaboonchai, W. (2014). Development and characterization of lutein-loaded SNEDDS for enhanced absorption in Caco-2 cells. Pharmaceutical Development and Technology, 19, 735-742.

O'Sullivan, L., Ryan, L., \& O'Brien, N. (2007). Comparison of the uptake and secretion of carotene and xanthophyll carotenoids by Caco-2 intestinal cells. British Journal of Nutrition, 98(1), 38-44.

Roberts, R. L., Green, J., \& Lewis, B. (2009). Lutein and zeaxanthin in eye and skin health. Clinics in Dermatology, 27(2), 195-201.

Satia, J. A., Littman, A., Slatore, C. G., Galanko, J. A., \& White, E. (2009). Long-term use of beta-carotene, retinol, lycopene, and lutein supplements and lung cancer risk: Results from the Vitamins And Lifestyle (VITAL) study. American Journal of Epidemiology, 170(3), 401-402.

Shanmugam, S., Baskaran, R., Balakrishnan, P., Thapa, P., Yong, C. S., \& Yoo, B. K. (2011). Solid self-nanoemulsifying drug delivery system (S-SNEDDS) containing phosphatidylcholine for enhanced bioavailability of highly lipophilic bioactive carotenoid lutein. European Journal of Pharmaceutics and Biopharmaceutics, 79(2), 250-257.

Shanmugasundaram, R., \& Selvaraj, R. (2011). Dietary lutein and fish oil interact to alter atherosclerotic lesions in a Japanese quail model of atherosclerosis. Journal of Animal Physiology and Animal Nutrition, 95(6), 762-770.

Tan, C., Xia, S., Xue, J., Xie, J., Feng, B., \& Zhang, X. (2013). Liposomes as vehicles for lutein: Preparation, stability, liposomal membrane dynamics, and structure. Journal of Agricultural and Food Chemistry, 61(34), 8175-8184.

Villwock, V. K., Eliasson, A.-C., Silverio, J., \& BeMiller, J. N. (1999). Starch-lipid interactions in common, waxy, ae du, and ae su2 maize starches examined by differential scanning calorimetry 1. Cereal Chemistry, 76(2), 292-298.

Vishwanathan, R. (2010). Lutein and zeaxanthin: Role as macular pigment and factors that control bioavailability from egg yolks and nanoemulsions.

Wong, K.-S., Kubo, A., Jane, J.-1., Harada, K., Satoh, H., \& Nakamura, Y. (2003). Structures and properties of amylopectin and phytoglycogen in the endosperm of sugary-1 mutants of rice. Journal of Cereal Science, 37(2), 139-149.

Xia, F., Hu, D., Jin, H., Zhao, Y., \& Liang, J. (2012). Preparation of lutein proliposomes by supercritical anti-solvent technique. Food Hydrocolloids, 26(2), 456-463. 